\section{Highlights of the San Antonio Breast Cancer Symposium 2015: part 1}

John R Benson ${ }^{*, 1} \&$ Ismail Jatoi²

\section{Future
ONCOLOGY}

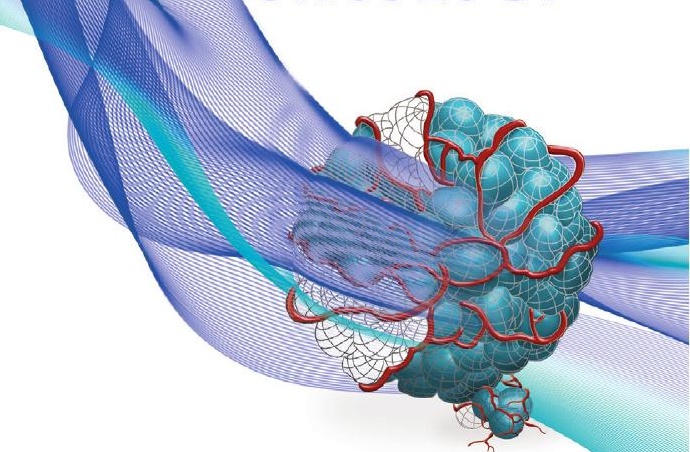

\section{8th San Antonio Breast Cancer Symposium, San Antonio, TX, USA, 8-12 December 2015}

The 38th annual San Antonio Breast Cancer Symposium (SABCS) was convened in San Antonio (TX, USA) on 8-12 December 2015. More than 7000 clinicians and scientists from around the world participated in the symposium, which featured a range of presentations and keynote talks pertaining to breast cancer screening, prevention, loco-regional and systemic therapies. This two-part report highlights a selection of important studies presented at this premier breast cancer event with part 1 focusing on radiotherapy treatment, translational approaches to immunotherapy and longer term cardiotoxicity from anti-HER2 therapies. The second part of this report will discuss a range of topics including de-escalation of chemotherapy regimens in luminal A tumors, the prognostic relevance of circulating tumor cells, the optimum treatment of triple-negative breast cancer and shifting attitudes to primary surgical treatment.

First draft submitted: 22 January 2016; Accepted for publication: 1 February 2016; Published online: 15 February 2016

In the first plenary lecture of the symposium, Jay Harris (Dana-Farber Cancer Institute, MA, USA) addressed critical decision-making pertaining to administration of radiotherapy (RT) in the context of breast-conserving surgery (BCS). He emphasized at the outset how a seminal meta-analysis by the Early Breast Cancer Trialists Collaborative Group (EBCTCG) in 2005 revealed that RT not only reduces local recurrence following either mastectomy or BCS but also confers a survival advantage [1]. However the postulated 4:1 ratio with one additional survivor at 15 years for every four local recurrences prevented at 5 years may be erroneous and it is now acknowledged that the primary end point for effect of RT should be 'any first recurrence', be this local or distant. The EBCTCG ratio has now been amended to one additional survivor at 20 years for every 1.5 first recurrence avoided at 10 years [2] Radiotherapy generally reduces any first recurrence proportionately by about one half with a corresponding mortality reduction of about $15 \%$ (one sixth). Harris pointed out that the survival benefit of RT is not mediated by reduction in local recurrence. Rates of local recurrence after BCS have fallen dramatically in recent years with a contemporaneous rate of about $2 \%$ at 5 years while within the NSABP trials there is a range of $3.5-13.3 \%$ local recurrence at 10 years [3]. This has been attributed to improvements in radiological/pathological evaluation in combination with more effective adjuvant systemic therapies (hormonal, chemotherapy and biological). These low rates of in-breast recurrence prompt the question of whether RT is necessary for all patients after BCS, some of whom derive small

\section{KEYWORDS}

- breast-conserving surgery

- mastectomy $\bullet$ radiation

treatment $\bullet$ San Antonio

- triple-negative cancer 
absolute benefits. Harris believes it is now appropriate to explore ways in which RT can be safely omitted in certain subgroups; the CALGB 9343 trial demonstrated that omission of RT after BCS was acceptable in patients aged $\geq 70$ years with small $(\leq 2 \mathrm{~cm})$, nonhigh-grade hormone receptor positive tumors without nodal involvement treated with endocrine therapy for whom 10-year mortality rates were only 5\% [4]. Many of these patients will have luminal A tumors and biological subtype is now recognized as a major determinant of local recurrence from studies of multivariate analysis [5]. Moreover, there is a convergence of risk factors for local recurrence after both BCS and mastectomy with younger age, tumor size (relative risk [RR]: 2.03) and node positivity (RR: 2.46) being highly predictive of local recurrence [6]. A new protocol has been proposed at Dana-Farber Cancer Institute/Massachusetts General Hospital in which women aged between 50 and 75 years with T1 N0 luminal A (Prosigna, WA, USA) tumors will be offered the option of hormonal therapy only without irradiation of the breast. Harris believes there are sufficient data from the UK START [7] and Ontario [8] trials to justify expansion of hypofractionation techniques which are associated with improved cosmesis, patient satisfaction and possibly overall survival (hazard ratio: $0.80 ; \mathrm{p}=0.04)$. Hypofractionation also reduces dose inhomogeneity $(<7 \%)$ and is cheaper and more convenient for patients. Indications for regional nodal irradiation (RNI) remain controversial with potential problems reconciling data from the Z0011 [9] and MA.20 trials [10]. Although patients in Z0011 did formally receive nodal irradiation, standard tangents capture a substantial proportion of level I/II nodes with evidence that fields were raised in Z0011 ('high tangents') to capture more nodes in up to $15 \%$ of patients. Moreover, this trial was not blinded and it appears that radiation oncologists were inclined to treat patients in the observation arm (no further axillary surgery) with high tangent RT to the axilla. These differential fields might partially account for similar outcomes in the two arms of the Z0011 trial. Both the MA.20 and EORTC [11] trials demonstrated small improvements in disease-free survival at 10 years from RNI with absolute differences in loco-regional and distant recurrence of 2.5 and $2.0 \%$, respectively in the MA.20 trial. It is unclear whether these benefits are due to irradiation of the supraclavicular/apical nodes, the internal mammary nodal irradiation or both. In the post-Z0011 era, nomograms can be used to estimate the probability of greater than two nodes positive with consideration of RNI.

In the Clinical Science Forum, Rehma Jagsi (University of Michigan Health System, MI, USA) emphasized that efficacy data from Z0011 cannot be extrapolated to other patient populations but some patients with low-volume metastases to the axilla can be managed with 'high tangent' or more formal axillary RT rather than axillary dissection to minimize morbidity (lymphedema). Higher risk patients with more extensive axillary tumor burden and aggressive biology should be considered for comprehensive nodal irradiation. Carolyn Taylor (EBCTCG) reminded the audience how radiotherapy techniques have changed with reduction in dosage to normal tissues and an overall risk for lung cancer and cardiac death of less than $1 \%$ for nonsmokers. The risks of heart disease and lung cancer in a nonsmoker aged 50 years are 0.1 and $0.5 \%$, respectively by age 80 years compared with 0.5 and $4.0 \%$ for smokers. The analysis by the EBCTCG found these elevated risks attenuate with cessation of smoking with much of the increased risk for lung cancer starting greater than 10 years after RT.

Several presentations focused on the tumor immune and stromal microenvironment and how signals from both the immune system and stromal cells can influence prognosis in triplenegative breast cancer (TNBC). Sherene Loi (Peter MacCallum Cancer Centre, Melbourne, Australia) examined the prognostic value of tumor-infiltrating lymphocytes (TiLs) in a pooled analysis of five randomized controlled trials involving almost 1000 patients with early-stage TNBC treated with anthracycline-based chemotherapy. TiLs are a surrogate for an adaptive immune response with both intratumoral and stromal TiLs (direct contact with and between tumor cells, respectively) adding significant information as a continuous variable to prognostic factors of age, tumor size and nodal status [12]. Those patients with node-negative breast cancer expressing $\geq 20 \%$ stromal TiLs have excellent 5-year survival estimates on Kaplan-Meier plots (92\%; 95\% CI: 87-97\%) and the effect of TiLs accords with a linear model. Moreover, there is a predominant influence of stromal over intratumoral TiLs based on likelihood ratio testing with the latter adding minimal further prognostic information to stromal TiLs. A net increment in TiLs of approximately $10 \%$ was associated with a $12 \%$ adjusted RR reduction in end points of recurrence and death. Christine Desmedt (Institute Jules Bordet, Brussels, Belgium) emphasized the 
need to standardize assessment of TiLs and correlate predefined cut-off values with conventional clinical/pathological indices. Although levels of TiLs are higher for TNBC and HER-positive tumors showing complete pathological response to neoadjuvant chemotherapy, the predictive value of TiLs remains unclear. In particular, for invasive lobular cancers which are characterized by lymphocytic infiltration, higher levels of TiLs have been found in tumors with adverse features estrogen/progesterone receptor-negative, high grade, high proliferation indices, node positivity, young age) and also the presence of specific mutations and copy number alterations. Thus the highest category of TiLs (>10\%) appears associated with a worse prognosis on univariate but not multivariate analysis.

Luc Dirix (Sint-Augustinus Hospital, Oosterveldlaan, Belgium) presented outcome data from the Phase Ib JAVELIN trial evaluating the anti-PD-L1 antibody avelumab in patients with locally advanced or metastatic breast cancer. This human IgG1 antibody binds PD-L1 and thereby inhibits interaction with PD-1 but leaves the PD-1/PD-L2 pathway intact [13]. Patients were unselected for PD-L1, hormone receptor/HER2 expression or molecular subtype and PD-L1 status was determined by immunohistochemistry. All 168 patients enrolled had a life expectancy of at least 3 months and remained on avelumab until progression. The drug was discontinued due to immune-related side effects in eight patients but safety and tolerability was deemed acceptable (two treatment-related deaths). The overall response rate was only $4.8 \%$, but among the eight responders, five $(62.5 \%)$ had TNBC. Of note, higher PD-L1 expression $(\geq 10 \%)$ by immune cells within the tumor was associated with improved response compared with immune cell-negative tumors (4/12 [33.3\%] vs 3/124 [2.4\%]).

The MANTICORE study from Canada aimed to determine whether standard therapeutic agents employed for treatment of established trastuzumab-related cardiotoxicity could be used as a preventive strategy. Trastuzumab causes left ventricular dysfunction in about $20 \%$ and potentially lethal heart failure in $1-5 \%$ of patients. In a three-arm double-blinded multicenter study, patients with HER2-positive early breast cancer were randomized 1:1:1 to receive placebo, an angiotensin-converting enzyme inhibitor (perindopril) or a $\beta$-blocker (bisoprolol) with no significant differences in cardiovascular characteristics (smoking habits, Type II diabetes, dyslipidemia, alcohol consumption). Edith Pituskin (Alberta Cancer Research Institute, Alberta, Canada) presented an interim analysis (99 patients) of this study for which the primary outcome was change in left ventricular end-diastolic volume on cardiac MRI. Neither an angiotensin-converting enzyme inhibitor nor a $\beta$-blocker $(\mathrm{p}=0.001)$ could prevent trastuzumab-induced left ventricular remodeling and therefore this study was negative for the primary end point. Nonetheless, it is important to identify early changes in left ventricular geometry and function although these appear irreversible. There was evidence that prophylactic use of a $\beta$-blocker offered protection from declines in left ventricular ejection fraction and could minimize interruptions to trastuzumab therapy. Dennis Slamon (UCLA Medical Center, CA, USA) updated the BCIRG-006 trial comparing doxorubicin plus cyclophosphamide followed by docetaxel alone (AC-T) or combined with trastuzumab (AC-TH) with docetaxel, carboplatin and trastuzumab (TCH) in HER2-positive earlystage breast cancer. There remains a sustained and significant survival advantage for Herceptincontaining regimens (TCH; AC-TH) compared with AC-T at 10 years. However, there is loss of cardiac function (>10\% decrease left ventricular ejection fraction) with fivefold higher rates of cardiac failure for AC-TH compared with TCH.

\section{Financial \& competing interests disclosure}

JR Benson was on the Planning Committee and I Jatoi on the Executive Committee for the 2015 San Antonio Breast Cancer Symposium. The authors have no other relevant affiliations or financial involvement with any organization or entity with a financial interest in or financial conflict with the subject matter or materials discussed in the manuscript apart from those disclosed.

No writing assistance was utilized in the production of this manuscript.

\section{References}

1 Clarke M, Collins R, Darby S et al. Effects of radiotherapy and of differences in the extent of surgery for early breast cancer on local recurrence and 15-year survival: an overview of the randomised trials. Lancet 366(9503), 2087-2106 (2005).

2 Early Breast Cancer Trialists' Collaborative Group (EBCTCG). Effect of radiotherapy after breast-conserving surgery on 10 -year recurrence and 15-year breast cancer death: meta-analysis of individual patient data for 10801 women in 17 randomised trials. Lancet 378(9804), 771-784 (2011). 
3 Anderson SJ, Wapnir I, Dignam JJ et al. Prognosis after ipsilateral breast tumor recurrence and locoregional recurrences in patients treated by breast-conserving therapy in five national surgical adjuvant breast and bowel project protocols of node-negative breast cancer. J. Clin. Oncol. 27(15), 2466-2473 (2009).

4 Hughes KS, Schnaper LA, Bellon JR et al. Lumpectomy plus tamoxifen with or without irradiation in women 70 years of age or older with early breast cancer. J. Clin. Oncol. 31(19), 2377-2381 (2013).

5 Nguyen PL, Taghian AG, Katz MS et al. Breast cancer subtype approximated by estrogen receptor, progesterone receptor, and HER-2 is associated with local and distant recurrence after breast-conserving therapy. J. Clin. Oncol. 26(14), 2373-2378 (2008).

6 Arvold ND, Taghian AG, Niemierko A et al. Age, breast cancer subtype approximation, and local recurrence after breast-conserving therapy. J. Clin. Oncol. 29(29), 3885-3891 (2011).

7 Haviland JS, Owen JR, Dewar JA et al. The UK Standardisation of Breast Radiotherapy (START) trials of radiotherapy

hypofractionation for treatment of early breast cancer: 10-year follow-up results of two randomized controlled trials. Lancet Oncol. 14(11), 1086-1094 (2013).

8 Whelan TJ, Pignol JP, Levine MN et al. Long-term results of hypofractionated radiation therapy for breast cancer. $N$. Engl. J. Med. 362(6), 513-520 (2010).

9 Guiliano AE, Hunt KV, Ballman KK et al. Axillary dissection vs no axillary dissection in women with invasive breast cancer and sentinel node metastases: a randomized clinical trial. JAMA 305(6), 569-575 (2011).

10 Whelan TJ, Olivotto IA, Parulekar WR et al. Regional nodal irradiation in early-stage breast cancer. N. Engl. J. Med. 373(19) 307-316 (2015).

11 Poortmans PM, Collette S, Kirkove C et al. Internal mammary and medial supraclavicular irradiation in breast cancer. N. Engl. J. Med. 373(4), 317-327 (2015).

12 Loi S, Sirtaine N, Piette F et al. Prognostic and predictive value of tumor-infiltrating lymphocytes in a Phase III randomized adjuvant breast cancer trial in node-positive breast cancer comparing the addition of docetaxel to doxorubicin with doxorubicinbased chemotherapy: BIG 02-98. J. Clin. Oncol. 31(7), 860-867 (2013).

13 Disis ML, Patel MR, Pant $S$ et al. Avelumab (MSB0010718C), an anti-PD-L1 antibody, in patients with previously treated, recurrent or refractory ovarian cancer: a Phase Ib, open-label expansion trial. J. Clin. Oncol. 33(Suppl.), Abstract 5509 (2015). 